\title{
Gadolinium chloride suppresses acute rejection and induces tolerance following rat liver transplantation by inhibiting Kupffer-cell activation
}

\author{
YAKUN WU ${ }^{1,2}$, YUNBING WANG $^{2}$, MIN LI $^{1,2}$, XIAOLI YANG ${ }^{1,2}$, JIANPING GONG $^{2}$ and WEI ZHANG ${ }^{1}$ \\ ${ }^{1}$ Department of Hepatobiliary Surgery, Suining Central Hospital, Suining, Sichuan 629000; \\ ${ }^{2}$ Department of Hepatobiliary Surgery, The Second Affiliated Hospital, \\ Chongqing Medical University, Chongqing 400010, P.R. China
}

Received November 10, 2013; Accepted May 7, 2014

DOI: $10.3892 /$ etm.2014.2015

\begin{abstract}
The aim of the present study was to investigate the mechanism by which gadolinium chloride $\left(\mathrm{GdCl}_{3}\right)$ inhibits Kupffer cell (KC) activation and its ability to suppress acute rejection and induce tolerance following liver transplantation in rats. Rats were randomly divided into control, liver transplantation with $\mathrm{GdCl}_{3}$ pretreatment and liver transplantation with normal saline pretreatment groups. The survival rate, liver function, hepatic pathological histology, cytokine levels in the liver and bile, activity of nuclear factor $\kappa$-light-chain-enhancer of activated $\mathrm{B}$ cells $(\mathrm{NF}-\kappa \mathrm{B})$ in $\mathrm{KCs}$, and expression levels of membranous molecules on the $\mathrm{KCs}$ were observed. It was identified that the one-month survival rate in the $\mathrm{GdCl}_{3}$ group was significantly higher compared with that in the saline group $(\mathrm{P}<0.05)$. The liver function in the $\mathrm{GdCl}_{3}$ group gradually recovered following transplantation surgery; however, it progressively deteriorated in the saline group. There were minor changes of hepatic pathological histology in the $\mathrm{GdCl}_{3}$ group, whereas changes typical of acute rejection occurred in the saline group. In the $\mathrm{GdCl}_{3}$ group, the levels of interferon $\gamma$ and interleukin (IL)-2 were significantly lower whereas the levels of IL-10 were significantly higher compared with those in the control and saline groups (all $\mathrm{P}<0.01$ ). The IL-4 levels in the $\mathrm{GdCl}_{3}$ and control groups were similar. The activity of $\mathrm{NF}-\kappa \mathrm{B}$ in the saline group was significantly higher compared with those in the control and $\mathrm{GdCl}_{3}$ groups $(\mathrm{P}<0.01)$. The expression levels of major histocompatibility complex-II, cluster of differentiation (CD)80 and CD86 on the $\mathrm{KC}$ membranes in the $\mathrm{GdCl}_{3}$ group was significantly lower compared with those in the control group $(\mathrm{P}<0.05)$; however,
\end{abstract}

Correspondence to: Dr Wei Zhang, Department of Hepatobiliary Surgery, Suining Central Hospital, 127 Desheng Road, Chuanshan, Suining, Sichuan 629000, P.R. China

E-mail: zhangweimmr@126.com

Key words: gadolinium chloride, Kupffer cells, liver transplantation these membranous proteins were highly expressed in the saline group. These data indicate that $\mathrm{GdCl}_{3}$ efficiently inhibits the immunological activity of KCs, suppresses acute rejection and induces tolerance following liver allograft transplantation in rats.

\section{Introduction}

Postoperative acute rejection (AR) in clinical liver transplantation is a major cause of early allograft dysfunction and acute function failure. A number of studies suggest that the inhibitory effect of gadolinium chloride $\left(\mathrm{GdCl}_{3}\right)$ against Kupffer cell (KCs) activation shows potential as a protective intervention in rat models of in vivo hepatic reperfusion injury and isolated perfused livers (1-3). It has also been shown that treatment of liver ischemia-reperfusion injury with $\mathrm{GdCl}_{3}$ reduces the mortality rate, attenuates neutrophil infiltration and decreases myeloperoxidase activity, improves hepatic function, reduces platelet accumulation in cold perfused livers, and prevents apoptosis of sinusoidal endothelial cells (4). Reduced free radical formation, lipid peroxidation, and parenchymal necrosis following $\mathrm{GdCl}_{3}$ administration have also been reported (5). In addition, treatment with $\mathrm{GdCl}_{3}$ diminishes the production of reactive oxygen species and the liberation of inflammatory mediators and inhibits the expression of adhesion molecules (6).

Thus, the identification of a treatment that is able to specifically inhibit AR and induce immune tolerance is urgent and essential. The activation of donor KCs is closely correlated with the occurrence of AR with intense phagocytosis, high expression levels of membranous molecules, clearly demonstrated antigen presentation, and the secretion of numerous cytokines that participate in the immune reaction (7-9). Therefore, by blocking the immune activity of KCs, AR may effectively be prevented and inhibited following surgery. In the present study, $\mathrm{GdCl}_{3}$ was used to inhibit the immune function of KCs and the depressant function of this treatment on liver transplantation AR was investigated, with the aim of investigating the underlying mechanism and providing experimental evidence for the successful inhibition of postoperative AR in clinical liver transplantation. 
Table I. Survival rates of postoperative rats in the control, $\mathrm{GdCl}_{3}$ and saline groups.

\begin{tabular}{lccccccc}
\hline & \multicolumn{7}{c}{ Postoperative survival rates, \% } \\
\cline { 2 - 7 } Group & 1 day & 2 days & 3 days & 4 days & 5 days & 7 days & 1 month \\
\hline Control & 100 & 100 & 100 & 100 & 100 & 100 & 99 \\
$\mathrm{GdCl}_{3}$ & 100 & 100 & 100 & 100 & 100 & 100 & $86^{\mathrm{a}}$ \\
Saline & 100 & 100 & 93 & 87 & 87 & 80 & 47 \\
\hline
\end{tabular}

${ }^{\mathrm{a}} \mathrm{P}<0.01$ vs. saline group.

\section{Materials and methods}

Experimental animals and treatment. Male Lewis (LEW) and Brown Norway (BN) rats (210-250 g), used as donors and recipients, respectively, were purchased from the animal research center of Chongqing Medical University (Chongqing, China). All the animals were housed in the animal care facility and received humane care in accordance with the National Institutes of Health guidelines for animal research and the legal requirements in China. The study was approved by the Ethics committee of the Second Affiliated Hospital of Chongqing Medical University (Chongqing, China). All the rats were randomly divided into three groups. The control group comprised $\mathrm{BN}$ rats $(\mathrm{n}=10)$ that underwent exploratory laparotomy. The $\mathrm{GdCl}_{3}$ group (liver transplantation with $\mathrm{GdCl}_{3}$ pretreatment group) used LEW $(\mathrm{n}=15)$ and $\mathrm{BN}$ rats $(\mathrm{n}=15)$ and $2 \mathrm{~g} / \mathrm{l} \mathrm{GdCl}_{3}$ solution $(7 \mathrm{mg} / \mathrm{kg}$ of body weight; Sigma-Aldrich, St. Louis, MO, USA) was injected via the vena caudalis into the donor with two days of continuous administration. On the third day, the donor liver was transplanted to the recipient. The saline group (liver transplantation with normal saline pretreatment group) used LEW $(n=15)$ and $B N$ rats $(n=15)$, and all the operative procedures were just as in the $\mathrm{GdCl}_{3}$ group, with the exception of using an identical volume of normal saline instead of $\mathrm{GdCl}_{3}$ solution.

Construction of models of liver transplantation. Orthotopic liver transplantations were performed from the LEW to BN rats using Kamada's method with a few modifications (10). The infrahepatic vena cava and the portal vein were linked with cuffs. The suprahepatic vena cava was inosculated with suture. A stent tube of the common bile duct was inserted into the common bile duct and the opening of the stent tube was left outside the body and used for collecting bile.

Analysis of plasma liver function markers and histopathological changes. The recipients were humanely sacrificed for histological inspection seven days post-surgery. The liver tissues were fixed in $100 \mathrm{~g} / \mathrm{l}$ neutral formalin solution, embedded in paraffin wax and the sections were stained with hematoxylin and eosin to assess the morphological changes. The blood of rats was obtained through the caudal vein. Plasma liver function markers, specifically, serum alanine aminotransferase (ALT), aspartate transaminase (AST) and total bilirubin (TB), were measured with an automatic biochemical meter (Beckman CX7; Beckman Coulter, Brea, CA, USA).

Reverse transcription polymerase chain reaction (RT-PCR) for cytokine mRNA analysis. RNA was extracted from the liver tissue with a TRIzol reagent kit (Life Technologies, Carlsbad, CA, USA). RT-PCR was performed using an RT-PCR kit (Roche, Los Angeles, CA, USA). The cDNA produced was used for the amplification of interferon (IFN)- $\gamma$, IL-2, IL-4, IL-10 and $\beta$-actin, respectively (primers were made by the Shanghai Biochemical Products Factory, Shanghai, China). Specific primer sequences of IL-10, IL-2, IL-4, IFN- $\gamma$ and $\beta$-actin were as follows: IL-10, forward: 5'-CCA AGC TTA TCG GAA ATG-3', and reverse: 5'-CAC TTG TAA ATC TTT CTT CGGG-3'; IL-2, forward: 5'-TAG TGG CTG TCG AGA AGC TGC3', and reverse: 5'-GGC GTC TTT CAT AGA CAG G-3'; IL-4, forward: 5'-CAT GGT CCG AGA TGT GCA ACT GGC-3', and reverse: 5'-CGG GCT CAG CAA CTC CAG C-3'; IFN- $\gamma$, forward: 5'-CCA CGA GGA ATT CTA CGC CCT GGGC-3', and reverse: 5'-AAG CTT GGG GAA CAG GTA GG-3'; $\beta$-actin, forward: 5'-CAT TGT GAT GGA CTC CGG AG-3', and reverse: 5'-CTG CCG GTC CAG TAG TATA-3'. The PCR conditions were 30 cycles of denaturation at $94^{\circ} \mathrm{C}$ for $60 \mathrm{sec}$, annealing at $58^{\circ} \mathrm{C}$ for $60 \mathrm{sec}$ and extension at $72^{\circ} \mathrm{C}$ for $60 \mathrm{sec}$, and finally an extension at $72^{\circ} \mathrm{C}$ for $7 \mathrm{~min}$. Agarose gel electrophoresis was used to separate the products of PCR. Ethidium bromide staining, a gelatin imaging system and figure analysis system (GelDoc 2000; Bio-Rad, Hercules, CA, USA) were also used for observing and semiquantitatively counting their relative quantities, expressed as relative optical density (ROD) with normalization to $\beta$-actin.

ELISA for analysis of cytokines in bile. On the seventh day after transplantation, bile was collected in order to measure the expression levels of IFN- $\gamma$ and IL-4 with an ELISA reagent kit (Beijing Dingguo Changsheng Biotech Co. Ltd., Beijing, China), following the manufacturer's instructions.

Isolation of KCs. KCs were isolated using collagenase digestion and differential centrifugation using Percoll. KCs were collected and cultured in plates with RPMI-1640 solution at $37^{\circ} \mathrm{C}$ in the presence of $5 \% \mathrm{CO}_{2}$. Non-adherent cells were removed after $6 \mathrm{~h}$ by replacing the buffer. The KCs were regulated to a density of $1 \times 10^{6}$ cells/well. The purity and viability of the cells were $>90$ and $>95 \%$, respectively. Next, 

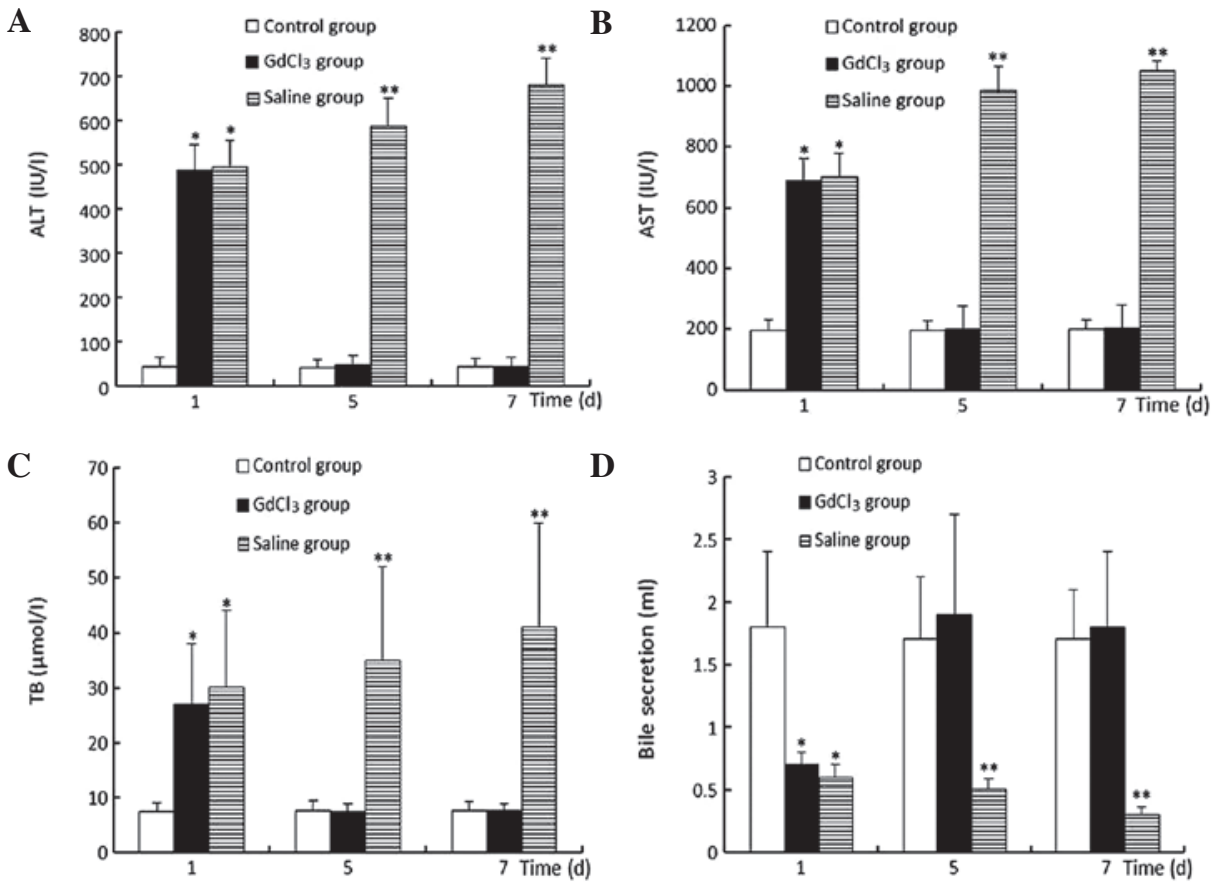

Figure 1. Plasma liver function markers level in the control, $\mathrm{GdCl}_{3}$ and saline groups at 1,5 and 7 days after surgery. (A) ALT, (B) AST and (C) TB levels in the serum. (D) Secretion of bile in the three groups. ${ }^{*} \mathrm{P}<0.01$ vs. control group;"* $\mathrm{P}<0.01$ vs. control and $\mathrm{GdCl}_{3}$ groups. ALT, alanine aminotransferase; AST, aspartate transaminase; TB, total bilirubin.

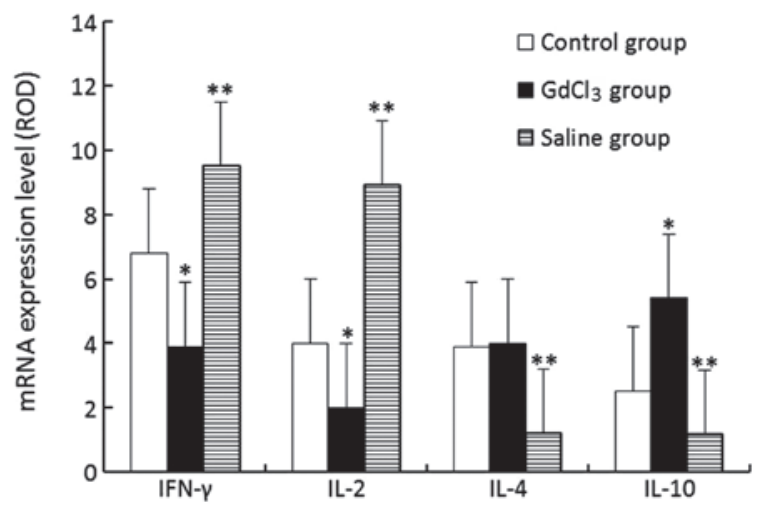

Figure 2. mRNA expression levels of hepatocellular cytokines in the control, $\mathrm{GdCl}_{3}$ and saline groups. ${ }^{*} \mathrm{P}<0.05$ vs. control group; ${ }^{* * *} \mathrm{P}<0.01$ vs. control and $\mathrm{GdCl}_{3}$ groups. ROD, relative optical density; IFN, interferon; IL, interleukin.

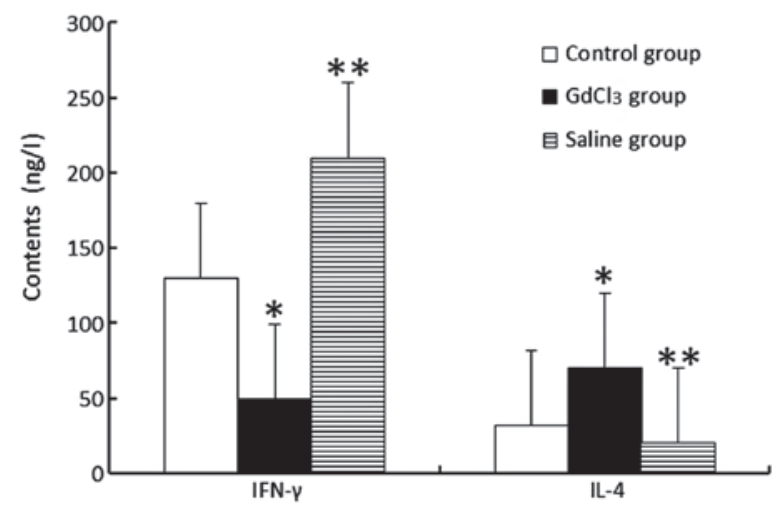

Figure 3. ELISA analytical results of cytokines in the bile. ${ }^{*} \mathrm{P}<0.05$ vs. control group; ${ }^{* * *} \mathrm{P}<0.01$ vs. the respective control and $\mathrm{GdCl}_{3}$ groups. IFN, interferon; IL, interleukin. the morphological characteristics of the KCs were observed under a phase contrast microscope (BX51; Olympus, Tokyo, Japan).

Detection of nuclear factor $\kappa$-light-chain-enhancer of activated $B$ cells $(N F-\kappa B)$ in KCs. For NF- $\kappa B$ activity analysis, $\mathrm{KCs}$ were harvested and lysed at $24 \mathrm{~h}$ after liver transplantation, and nuclear proteins were extracted using an Active Motif Nuclear Extract kit (Active Motif, Carlsbad, CA, USA). The relative activity of NF- $\mathrm{BB}$ was represented by the optical density value of colorimetric analysis. The DNA-binding activity of NF- $\kappa \mathrm{B}$ was determined by ELISA using the TransAM ${ }^{\circledR}$ NF- $\kappa B$ p65 Family kit (Active Motif), according to the manufacturer's instructions. In brief, nuclear extract was transferred into a 96-well plate coated with NF-кB p65 consensus oligonucleotides. Subsequently, the NF- $\kappa \mathrm{B}$ p65 protein bound to the target sequence was detected by primary rabbit anti-rat p65 antibody (Santa Cruz Biotechnology Inc., Santa Cruz, CA, USA) and a goat anti-rabbit horseradish peroxidase-conjugated secondary antibody (Santa Cruz Biotechnology Inc.). Absorbance was quantified by spectrophotometry (DR 5000 spectrophotometer, Hach Company, Indiana, USA) at $595 \mathrm{~nm}$ as a relative measure of protein-bound NF- $\kappa \mathrm{B}$ p65.

Membranous molecules on KCs. A monoclonal antibody against major histocompatibility complex (MHC)-II, cluster of differentiation (CD)80 or CD86 (Santa Cruz Biotechnology Inc., Santa Cruz, CA, USA) combined with fluorescein isothiocyanate was added to the liquid suspension of KCs that was acquired previously and the mixture was incubated for $30 \mathrm{~min}$. A flow cytometer (FC500 MPL; Beckman 


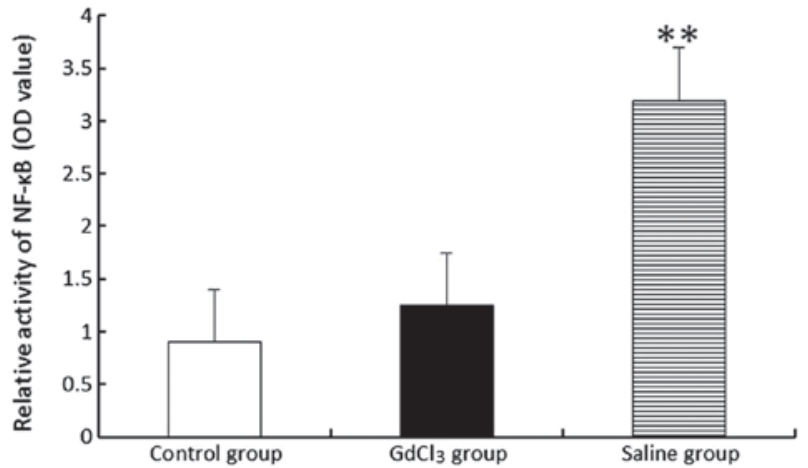

Figure 4. Relative activity of $\mathrm{NF}-\kappa \mathrm{B}$ represented by the optical density (OD) value in the control, $\mathrm{GdCl}_{3}$ and saline groups. ${ }^{* *} \mathrm{P}<0.01$ vs. control and $\mathrm{GdCl}_{3}$ groups. $\mathrm{NF}-\kappa \mathrm{B}$; nuclear factor $\kappa$-light-chain-enhancer of activated $\mathrm{B}$ cells.

Coulter) was used to assay the positive cells and the mean fluorescence intensity.

Data analysis. All data are expressed as the mean \pm standard deviation. Statistical analyses were performed with SPSS software, version 9.0 (SPSS Inc., Chicago, IL, USA). Analysis of variance with Fisher's protected least significant difference post hoc analysis and Student's test was used to identify significant differences between the groups. $\mathrm{P}<0.05$ was considered to indicate a statistically significant difference.

\section{Results}

Survival condition of postoperative rats. In the initial two days following surgery, all rats in the $\mathrm{GdCl}_{3}$ and saline groups survived, and no difference in the quality of life was identified. From the third day following surgery, differences appeared between the groups. Food intake and energy levels gradually recovered to pre-operative levels in the $\mathrm{GdCl}_{3}$ group; however, in the saline group, these representations deteriorated. From the seventh day following surgery, the difference became increasingly evident. All the rats in the $\mathrm{GdCl}_{3}$ group survived and their general condition recovered to nearly normal levels. However, in the saline group, the rats presented clear occurrence of ascites, jaundice and even mortality. After the first month following surgery, the survival rates in the $\mathrm{GdCl}_{3}$ and saline groups were 86 and $47 \%$, respectively, and the difference was statistically significant $(\mathrm{P}<0.01$; Table I).

Histopathological changes of liver. Under a light microscope, the hepatocytes in the $\mathrm{GdCl}_{3}$ group presented moderate vacuolar degeneration and edema and a small number of inflammatory cells were observed to be infiltrating the portal area. However, in the saline group, the hepatocytes presented severe edema and large-area necrosis and large quantities of infiltrating inflammatory cells (not shown).

Plasma liver function markers. In the $\mathrm{GdCl}_{3}$ group, on the first day following surgery, the ALT, AST and TB levels were clearly elevated and the volume of bile secreted was decreased compared with those in the control group $(\mathrm{P}<0.05)$. Notably, from the second day after surgery, the

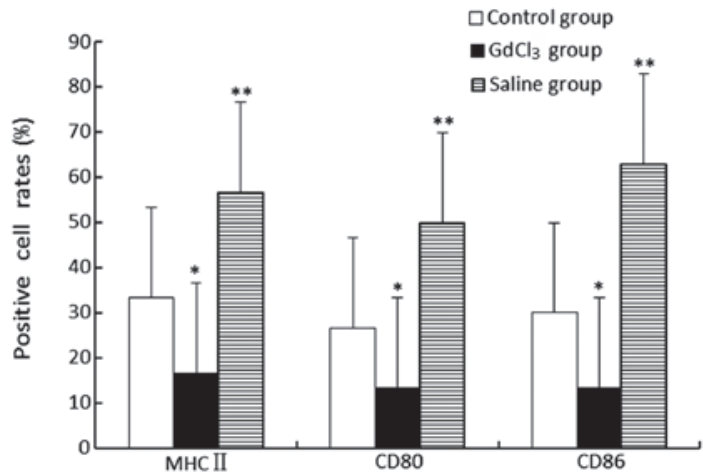

Figure 5. Expression levels of MHC-II, CD80 and CD86 in the control, $\mathrm{GdCl}_{3}$ and saline groups. ${ }^{*} \mathrm{P}<0.05$ vs. control group; ${ }^{* *} \mathrm{P}<0.01$ vs. respective control and $\mathrm{GdCl}_{3}$ groups. MHC-II, major histocompatibility complex class II; CD, cluster of differentiation.

aforementioned markers gradually recovered and by the fifth day after surgery, the markers were restored to normal levels. However, in the saline group, the aforementioned liver function markers were progressively aggravated, increasing to maximum levels on the seventh day following surgery (Fig. 1).

RT-PCR assay of hepatocellular cytokines. The expression levels of IFN- $\gamma$ and IL- 2 mRNA in the $\mathrm{GdCl}_{3}$ group were evidently lower compared with those in the control group $(\mathrm{P}<0.05)$; however, the IL-10 level in the $\mathrm{GdCl}_{3}$ group was higher than that in the control group $(\mathrm{P}<0.05)$ and the IL-4 levels showed no differences between the $\mathrm{GdCl}_{3}$ and control groups. The results for the saline group displayed an opposite trend to those in the $\mathrm{GdCl}_{3}$ group (Fig. 2).

ELISA analytical results of cytokines in bile. In terms of the ELISA analysis of bile, the concentration of IFN- $\gamma$ in the $\mathrm{GdCl}_{3}$ group was markedly lower compared with that in the control group $(\mathrm{P}<0.05)$. However, the IL-4 level in the $\mathrm{GdCl}_{3}$ group increased compared with that in the control group $(\mathrm{P}<0.05)$. The changes observed in the saline groups were completely opposite to those in the $\mathrm{GdCl}_{3}$ group (Fig. 3).

$N F-\kappa B$ activity. The activity of $\mathrm{NF}-\kappa \mathrm{B}$ was not revealed to differ between the $\mathrm{GdCl}_{3}(1.25 \pm 0.63)$ and control $(0.91 \pm 0.62)$ groups. However, $\mathrm{NF}-\kappa \mathrm{B}$ demonstrated an increased activity level in the saline $(3.21 \pm 0.65)$ group compared with those in the control and $\mathrm{GdCl}_{3}$ groups $(\mathrm{P}<0.01 ;$ Fig. 4$)$.

Expression levels of membranous molecules on KCs. The expression levels of MHC-II, CD80 and CD86 in the $\mathrm{GdCl}_{3}$ group were revealed to be lower compared with those in the control and saline groups $(\mathrm{P}<0.05)$. Additionally, the saline group highly expressed these three molecules $(\mathrm{P}<0.01 ;$ Fig. 5).

\section{Discussion}

$\mathrm{KCs}$, which are the resident macrophages of the liver, not only exert phagocytosis but also excrete significant amounts of pro-inflammatory and anti-inflammatory cytokines. The understanding of the contribution of KCs to the inflammatory 
response is likely to help in developing immunological and pharmacological strategies aimed at attenuating the excessive cytokine secretion and decreasing immune damage. Although the role of $\mathrm{KCs}$ in directly inducing liver transplantation (LT) tolerance has been reported $(7,11)$, immune tolerance by inhibition of $\mathrm{KCs}$ has been highlighted in numerous recent studies (11-13). A number of scholars in China and around the world have demonstrated that $\mathrm{GdCl}_{3}$ selectively reduces the number of KCs and their functions in experiments, through an unclear mechanism $(3,4,14)$. Based on this point, if $\mathrm{GdCl}_{3}$ was used in a $\mathrm{KC}$-associated study, the results may be beneficial in explaining the mechanism of hepatic postoperative acute rejection. In the present study, the aim was to explore the mechanism of the function of $\mathrm{GdCl}_{3}$ with regard to $\mathrm{KCs}$ and LT rejection and tolerance, and the following was concluded.

The $\mathrm{KCs}$ in the $\mathrm{GdCl}_{3}$ group represented a non-activated state with decreased apophysis on the cell surface, lower function of the organelle, and decreased expression of the MHC-II molecule. These observations indicate that $\mathrm{GdCl}_{3}$ treatment may inhibit or obstruct phagocytosis, absorption, antigen presentation and the combination capacity of the MHC of KCs with antigens or extraneous materials. In this way, immune rejection may be effectively prevented.

Activated $\mathrm{T}$ cells are critical in the immune response, and their activation requires two types of signal stimulus (from the combination of the T-cell receptor and the peptide-MHC and from co-stimulated molecules) (15). As identified in the $\mathrm{GdCl}_{3}$ group, the expression levels of co-stimulated molecules (CD80 and CD86) on the KCs decreased. A possible mechanism may be that by downregulating the expression of co-stimulated molecules on $\mathrm{KCs}$, the activation of $\mathrm{T}$ lymphocytes at the upper stream was prohibited.

The activation of $\mathrm{NF}-\kappa \mathrm{B}$ plays a controversial role due to its dual action in the induction of both protective and pro-inflammatory genes. However, it is generally recognized that this dichotomy of $\mathrm{NF}-\kappa \mathrm{B}$ promoting the hepatic inflammatory response and also protecting against it reflects the expression of $\mathrm{NF}-\kappa \mathrm{B}$-dependent genes in different hepatic cell populations (16). In the present study, it was identified that the activation of NF- $\kappa \mathrm{B}$ in the group pretreated with $\mathrm{GdCl}_{3}$ was lower compared with that in the saline group. Thus, inhibiting the $\mathrm{NF}-\kappa \mathrm{B}$ activity in KCs may be considered as a route of inducing immune tolerance.

$\mathrm{Th} 0$ is able to differentiate into two subsets of helper $\mathrm{T}$ cells (Th1 and Th2), depending on the cytokine stimulation and production. Furthermore, the role of KCs in this process cannot be ignored. It is well known that cytokines produced by these two subsets play pivotal roles in the modulation of the immune response. As reported in numerous studies, the Th1 cytokines, including IFN- $\gamma$ and IL-2, participate in the promotion of graft rejection (16-18). By contrast, an increased expression of the Th2 cytokines, including IL-10 and IL-4, may play a significant role in inducing an immune tolerance (19-24). This study also demonstrated that the IL-10 and IL-4 levels in the group pretreated with $\mathrm{GdCl}_{3}$ were evidently higher compared with the respective levels in the saline group; however, the IFN- $\gamma$ and IL-2 levels in the $\mathrm{GdCl}_{3}$ group were clearly lower compared with those in the saline group. These results may indicate that deviation from Th1 to Th2 may be a mechanism of immune tolerance.
In conclusion, $\mathrm{GdCl}_{3}$ efficiently inhibited the immunological activity of KCs and suppressed acute rejection following liver allograft transplantation in rats. In addition, it may be concluded that the immune tolerance induced by the inhibitory effect of $\mathrm{GdCl}_{3}$ on $\mathrm{KCs}$ was a combined action requiring the participation of numerous mechanisms. In further studies, new cytokines and regulative routes could be identified to explain this effect, and further studies may also be necessary for understanding the exact mechanism.

\section{Acknowledgements}

The study was supported by the National Natural Science Foundation of China (grant no. 81370580).

\section{References}

1. Rüttinger D, Vollmar B, Kempter B and Messmer K: Failure of Kupffer cell blockade to prevent disseminated intravascular coagulation in endotoxemic rats despite improved survival. Langenbecks Arch Surg 383: 75-80, 1998.

2. Vollmar B, Rüttinger D, Wanner GA, et al: Modulation of Kupffer cell activity by gadolinium chloride in endotoxemic rats. Shock 6: 434-441, 1996.

3. Dimitrios G, George P, Stavros I, et al. The protective effect of alpha-tocopherol and $\mathrm{GdCl}_{3}$ against hepatic ischemia/reperfusion injury. Surg Today 36: 450-456, 2006.

4. Sindram D, Porte RJ, Hoffman MR, et al: Synergism between platelets and leukocytes in inducing endothelial cell apoptosis in the cold ischemic rat liver: a Kupffer cell-mediated injury. FASEB J 15: 1230-1232, 2001.

5. Giakoustidis DE, Iliadis S, Tsantilas D, et al: Blockade of Kupffer cells by gadolinium chloride reduces lipid peroxidation and protects liver from ischemia/reperfusion injury. Hepatogastroenterology 50: 1587-1592, 2003.

6. Jahnke C, Mehrabi A, Golling M, et al: Evaluation of microperfusion disturbances in the transplanted liver after Kupffer cell destruction using $\mathrm{GdCl}_{3}$ : An experimental porcine study. Transplant Proc 38: 1588-1595, 2006.

7. Chen Y, Liu Z, Liang S, et al: Role of Kupffer cells in the induction of tolerance of orthotopic liver transplantation in rats. Liver Transpl 14: 823-836, 2008.

8. Chen Y, Liu H, Liu Z, et al: Blockade of inducible costimulator pathway to prevent acute rejection in rat liver transplantation. Am J Surg 198: 244-249, 2009.

9. Xie X, Ye Y,Zhou L, et al: Küpffer cells promote acute rejection via induction of Th17 differentiation in rat liver allografts. Transplant Proc 42: 3784-3792, 2010.

10. Delrivière L, Gibbs P, Kobayashi E, et al: Technical details for safer venous and biliary anastomoses for liver transplantation in the rat. Microsurgery 18: 12-18, 1998.

11. Chen GS and Qi HZ: Effect of Kupffer cells on immune tolerance in liver transplantation. Asian Pac J Trop Med 5: 970-972, 2012.

12. Tiegs $\mathrm{G}$ and Lohse $\mathrm{AW}$ : Immune tolerance: what is unique about the liver. J Autoimmun 34: 1-6, 2010.

13. You Q, Cheng L, Kedl RM and Ju C: Mechanism of T cell tolerance induction by murine hepatic Kupffer cells. Hepatology 48: 978-990, 2008.

14. Jones C, Badger SA Hoper M, et al: Hepatic cytokine response can be modulated using the Kupffer cell blocker gadolinium chloride in obstructive jaundice. Int J Surg 11: 46-51, 2013.

15. Lorenzo LP, Shatynski KE, Clark S, et al: Defective thymic progenitor development and mature T-cell responses in a mouse model for Down syndrome. Immunology 139: 447-458, 2013.

16. Llacuna L, Marí M Lluis JM, et al: Reactive oxygen species mediate liver injury through parenchymal nuclear factor-kappaB inactivation in prolonged ischemia/reperfusion. Am J Pathol 174: 1776-1785, 2009.

17. Zhao X, Boenisch O, Yeung M, et al: Critical role of proinflammatory cytokine IL-6 in allograft rejection and tolerance. Am J Transplant 12: 90-101, 2012.

18. Zhao J, Li P and Gao S: Effect of TGF-betal on the expression of IL-12, IL-15, IL-18, IL-4 and IL-10 in heart transplantation rejection in rats. J Huazhong Univ Sci Technolog Med Sci 27: 643-645, 2007. 
19. Chen Y, Yan T, Shi LJ, et al: Knockdown of interleukin-2 by shRNA-mediated RNA interference prolongs liver allograft survival. J Surg Res 159: 582-587, 2010.

20. Chen Y, Chen J, Liu Z, et al: Relationship between TH1/TH2 cytokines and immune tolerance in liver transplantation in rats. Transplant Proc 40: 2691-2695, 2008.

21. Liao W, Zeng F, Kang K, et al: Lipoxin A4 attenuates acute rejection via shifting $\mathrm{TH} 1 / \mathrm{TH} 2$ cytokine balance in rat liver transplantation. Transplant Proc 45: 2451-2454, 2013.

22. Feng JF, Chen F, Liu H, et al: Induction of immune tolerance by pre-infusion of apoptotic lymphocytes derived from peripheral blood of donor rats before liver transplantation. Minerva Chir 68: $183-189,2013$
23. Hu A, Li Q, Shi H, et al: Donor-derived bone marrow transfusion produces mixed chimerism and promotes a Th2 shift in Th1/Th2 balance in rat heterotopic small bowel transplantation. Dig Liver Dis 44: 988-994, 2012.

24. Lian ZR, Xu YF, Wang XB, et al: Suppression of histone deacetylase 11 promotes expression of IL-10 in Kupffer cells and induces tolerance following orthotopic liver transplantation in rats. J Surg Res 174: 359-368, 2012. 